\title{
Existence and exponential stability of solutions for transmission system with varying delay in $\mathbb{R}$
}

\author{
Salah Zitouni, Abdelouaheb Ardjouni, Khaled Zennir \\ AND RACHIDA AMIAR
}

\begin{abstract}
In the present paper we are going to consider in a one dimension bounded domain a transmission system with a varying delay. Under suitable assumptions on the weights of the damping and the delay terms, we prove the well-possedness and the uniqueness of solution using the semigroup theory. Also we show the exponential stability by introducing an appropriate Lyaponov functional.
\end{abstract}

\section{INTRODUCTION}

It is well known that the PDEs with time delay have been much studied during the last years and their results is by now rather developed. See [1], $[5,6,7,14,17,18,19]$. In the classical theory of delayed wave equations, several main parts are joined in a fruitful way, it is very remarkable that the damped wave equation with varying delays occupies a similar position and arise in many applied problems.

We consider the transmission problem with a varying delay term,

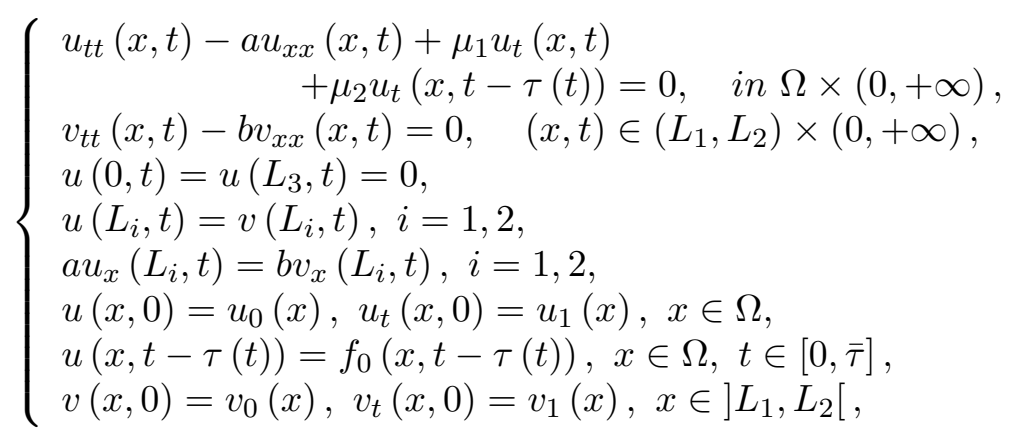

where $\left.0<L_{1}<L_{2}<L_{3}, \Omega=\right] 0, L_{1}[\cup] L_{2}, L_{3}\left[, a, b, \mu_{1}, \mu_{2}\right.$ are positive constants.

2000 Mathematics Subject Classification. Primary: 435B37; Secondary: 35L55.

Key words and phrases. Wave equation; transmission problem; varying delay term; exponential stability. 
We assume, that there exist positive constants $\tau_{0}, \bar{\tau}$ such that

$$
0<\bar{\tau}_{0} \leq \tau(t) \leq \bar{\tau}, \quad \forall t>0 .
$$

Moreover, we assume that

$$
\begin{aligned}
& \tau \in W^{2, \infty}([0, T]), \quad \forall T>0, \\
& \tau^{\prime}(t) \leq d<1, \quad \forall t>0,
\end{aligned}
$$

where $d$ is a positive constant.

To motivate our work, let us mention the work [16], when the authors studied well-posedness and exponential stability of a problem with structural damping and boundary delay in both cases $\mu>0$ and $\mu=0$ in a bounded and smooth domain, where $k_{2}=0$. The analogous problem with boundary feedback has been introduced and studied by Xu, Yung, Li [19] in one-space dimension using a fine spectral analysis and in higher space dimension by the authors [14]. The case of time-varying delay has been already studied in [15] in one space dimension and in general dimension, with a possibly degenerate delay, in [16]. Both these papers deal with boundary feedback.

This paper improves the results in [4]; for $\tau(t)=\tau$, under suitable assumptions on the weight of the damping and the weight of the delay, he prove the existence and the uniqueness of the solution using the semigroup theory. Also he show the exponential stability of the solution by introducing a suitable Lyaponov functional..

Without delay, system (1) has been investigated in [3]; for $\Omega=\left[0, L_{1}\right]$, the authors showed the well-posedness and exponential stability of the total energy. Muñoz Rivera and Oquendo [13] studied the wave propagations over materials consisting of elastic and viscoelastic components; that is,

$$
\begin{aligned}
\rho_{1} u_{t t}-\alpha_{1} u_{x x} & =0, x \in] 0, L_{0}[, t>0, \\
\rho_{2} v_{t t}-\alpha_{2} v_{x x}+\int_{0}^{t} g(t-s) v_{x x}(s) d s & =0, x \in] L_{0}, L[, t>0,
\end{aligned}
$$

with the boundary and initial conditions:

$$
\begin{aligned}
u(0, t) & =v(L, t), u\left(L_{0}, t\right)=v\left(L_{0}, t\right), t>0, \\
\alpha_{1} u_{x}\left(L_{0}, t\right) & =\alpha_{2} v_{x}\left(L_{0}, t\right)-\int_{0}^{t} g(t-s) v_{x}(s) d s, t>0,
\end{aligned}
$$

where $\rho_{1}$ and $\rho_{2}$ are densities of the materials and $\alpha_{1}, \alpha_{2}$ are elastic coefficients and $g$ is positive exponential decaying function. They showed that the dissipation produced by the viscoelastic part is strong enough to produce an exponential decay of the solution, no matter how small is its size. Ma and Oquendo [9] considered transmission problem involving two Euler-Bernoulli equations modeling the vibrations of a composite beam. By using just one boundary damping term in the boundary, they showed the global existence and decay property of the solution. Marzocchi et al [10] investigated a 1-D semi-linear transmission problem in classical thermoelasticity and showed 
that a combination of the first, second and third energies of the solution decays exponentially to zero, no matter how small the damping subdomain is. A similar result has sheen shown by Messaoudi and Said-Houari [12], where a transmission problem in thermoelasticity of type III has been investigated. See also Marzocchi et al [11] for a multidimensional linear thermoelastic transmission problem. The effect of the delay in the stability of hyperbolic systems has been investigated by many people. See for instance $[6,7]$. The aim of this article is to study effect of the varying delay in the stability of our system.

\section{WELL-POSEDNESS}

Using the semigroup theory, we prove the existence and uniqueness of solution of system (1). As in [14], let us introduce the following new variable

$$
z(x, \rho, t)=u_{t}(x, t-\tau(t) \rho) .
$$

Then, we obtain

(8) $\tau(t) z_{t}(x, \rho, t)+\left(1-\tau^{\prime}(t) \rho\right) z_{\rho}(x, \rho, t)=0, \quad$ in $\Omega \times(0,1) \times(0,+\infty)$.

Therefore, the first equation in problem (1) is become as

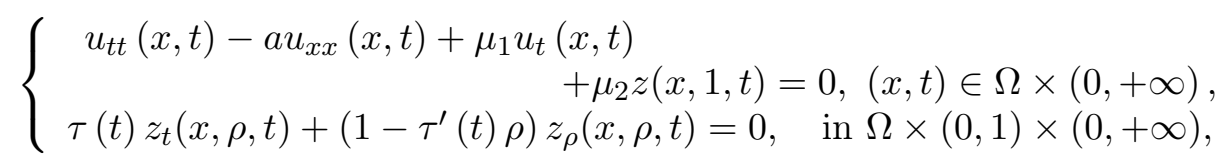

which can be written as

$$
\left\{\begin{array}{l}
U^{\prime}=\mathcal{A}(t) U \\
U(0)=\left(u_{0}, v_{0}, u_{1}, v_{1}, f_{0}(\cdot,-\cdot \tau)\right)
\end{array}\right.
$$

where the operator $\mathcal{A}(t)$ is given by

$$
\mathcal{A}(t)\left(\begin{array}{c}
u \\
v \\
\varphi \\
\psi \\
z
\end{array}\right)=\left(\begin{array}{c}
\varphi \\
\psi \\
a u_{x x}-\mu_{1} \varphi-\mu_{2} z(., 1) \\
b v_{x x} \\
\frac{\tau^{\prime}(t) \rho-1}{\tau(t)} z_{\rho}
\end{array}\right),
$$

with the domain

$$
D(\mathcal{A}(t))=\left\{(u, v, \varphi, \psi, z)^{T} \in \mathcal{H} ; z(., 0)=\varphi \text { on } \Omega\right\},
$$

where

$$
\mathcal{H}=X_{*} \times L^{2}(\Omega) \times L^{2}\left(L_{1}, L_{2}\right) \times L^{2}((\Omega) \times(0,1)),
$$

where the space $X_{*}$ is defined by

$$
\begin{aligned}
X_{*}= & \left\{(u, v) \in H^{1}(\Omega) \times H^{1}\left(L_{1}, L_{2}\right): u(0)=u\left(L_{3}\right)=0,\right. \\
& \left.u\left(L_{i}\right)=v\left(L_{i}\right), a u_{x}\left(L_{i}\right)=b v_{x}\left(L_{i}\right), i=1,2\right\} .
\end{aligned}
$$


Remark 2.1. Noting that the domain of $D(\mathcal{A})(t)$ is independent of the time $t$; i.e.,

$$
D(\mathcal{A}(t))=D(\mathcal{A}(0)), t>0 .
$$

Let

$$
U=(u, v, \varphi, \psi, z)^{T}, \quad \bar{U}=(\bar{u}, \bar{v}, \bar{\varphi}, \bar{\psi}, \bar{z})^{T} .
$$

We define the standard inner product in $\mathcal{H}$ as follows:

$$
\begin{aligned}
\langle U, \bar{U}\rangle_{\mathcal{H}}= & \int_{\Omega}\left\{\varphi \bar{\varphi}+a u_{x} \bar{u}_{x}\right\} d x+\int_{L_{1}}^{L_{2}}\left\{\psi \bar{\psi}+b v_{x} \overline{v_{x}}\right\} d x \\
& +\int_{\Omega} \int_{0}^{1} z(x, \rho) \bar{z}(x, \rho) d \rho d x .
\end{aligned}
$$

Using semigroup arguments by the literature, we can obtain a well-posedness result (see [8]).

Theorem 2.1. Assume that

(i) $D(\mathcal{A}(0))$ is a dense subset of $\mathcal{H}$,

(ii) $D(\mathcal{A}(t))=D(\mathcal{A}(0))$ for all $t>0$,

(iii) for all $t \in[0, T], \mathcal{A}(t)$ generates a strongly continuous semigroup on $\mathcal{H}$ and the family $\mathcal{A}=\{\mathcal{A}(t): t \in[0, T]\}$ is stable with stability constants $C$ and $m$ independent of $t$ (i.e. the semigroup $\left(S_{t}(s)\right)_{s \geq 0}$ generated by $\mathcal{A}(t)$ satisfies $\left\|S_{t}(s) u\right\|_{\mathcal{H}} \leq C e^{m s}\|u\|_{\mathcal{H}}$, for all $u \in \mathcal{H}$ and $s \geq 0$ ),

(iv) $\partial_{t} \mathcal{A}$ belongs to $L_{*}^{\infty}([0, T], B(D(\mathcal{A}(0)), \mathcal{H}))$, the space of equivalent classes of essentially bounded, strongly measurable functions from $[0, T]$ into the set $B(D(\mathcal{A}(0)), \mathcal{H})$ of bounded operators from $D(\mathcal{A}(0))$ into $\mathcal{H}$.

Then, problem (2) has a unique solution $U \in C([0, T], D(\mathcal{A}(0))) \cap C^{1}([0, T], \mathcal{H})$ for any initial datum in $D(\mathcal{A}(0))$.

Therefore, we will check the above assumptions for system (2).

Lemma 2.1. $D(\mathcal{A}(0))$ is dense in $\mathcal{H}$,

Proof. Let $\left(f, g, g_{1}, h_{1}, h_{2}\right)^{T} \in \mathcal{H}$ be orthogonal to all elements of $D(A(0))$, that is,

$$
\begin{aligned}
0 & =\left\langle(u, v, \varphi, \psi, z)^{T},\left(f, g, g_{1}, h_{1}, h_{2}\right)^{T}\right\rangle_{\mathcal{H}} \\
& =\int_{\Omega}\left\{\varphi g_{1}+a u_{x} f_{x}\right\} d x+\int_{L_{1}}^{L_{2}}\left\{\psi h_{1}+b v_{x} g_{x}\right\} d x+\int_{\Omega} \int_{0}^{1} z(x, \rho) h_{2}(x, \rho) d \rho d x,
\end{aligned}
$$

$\forall(u, v, \varphi, \psi, z)^{T} \in D(A(0))$. 
Taking $u=v=\varphi=\psi=0$ (then $\left.u_{x}=v_{x}=0\right)$ and $z \in D(\Omega \times(0,1))$. As $(0,0,0,0, z)^{T} \in D(A(0))$, we obtain

$$
\int_{\Omega} \int_{0}^{1} z(x, \rho) h_{2}(x, \rho) d \rho d x=0 .
$$

Since $D(\Omega \times(0,1))$ is dense in $L^{2}(\Omega \times(0,1))$, we deduce that $h_{2}=0$.

In the same way, by taking $u=v=\varphi=0$ (then $u_{x}=v_{x}=0$ ) and $\psi \in D\left(L_{1}, L_{2}\right)$. As $(0,0,0, \psi, 0)^{T} \in D(A(0))$, we obtain

$$
\int_{L_{1}}^{L_{2}} \psi h_{1} d x=0 .
$$

Since $D\left(L_{1}, L_{2}\right)$ is dense in $L^{2}\left(L_{1}, L_{2}\right)$, we deduce that $h_{1}=0$. Also for $u=v=0$ (then $\left.u_{x}=v_{x}=0\right)$ and $\varphi \in D(\Omega)$ we see that $g_{1}=0$. Therefore, for $(u, v) \in D\left(\Omega \times\left(L_{1}, L_{2}\right)\right)$ (then $\left.\left(u_{x}, v_{x}\right) \in D\left(\Omega \times\left(L_{1}, L_{2}\right)\right)\right)$ we obtain

$$
\int_{\Omega} a u_{x} f_{x} d x+\int_{L_{1}}^{L_{2}} b v_{x} g_{x} d x=0 .
$$

Since $D\left(\Omega \times\left(L_{1}, L_{2}\right)\right)$ is dense in $L^{2}\left(\Omega \times\left(L_{1}, L_{2}\right)\right)$, we deduce that $\left(f_{x}, g_{x}\right)=$ $(0,0)$ then $(f, g)=(0,0)$ because $(f, g) \in X_{*}$.

Assuming

$$
\mu_{2} \leq \sqrt{1-d} \mu_{1} .
$$

In order to deduce a well-posedness result, we define on $\mathcal{H}$ the time dependent inner product

$$
\begin{aligned}
& \left\langle(u, v, \varphi, \psi, z)^{T},(\bar{u}, \bar{v}, \bar{\varphi}, \bar{\psi}, \bar{z})^{T}\right\rangle_{\mathcal{H}} \\
& =\int_{\Omega}\left\{\varphi \bar{\varphi}+a u_{x} \bar{u}_{x}\right\} d x+\int_{L_{1}}^{L_{2}}\left\{\psi \bar{\psi}+b v_{x} \bar{v}_{x}\right\} d x \\
& +\xi \tau(t) \int_{\Omega} \int_{0}^{1} z(x, \rho) \bar{z}(x, \rho) d \rho d x,
\end{aligned}
$$

where $\xi$ is the positive constant satisfying

$$
\frac{\mu_{2}}{\sqrt{1-d}} \leq \xi \leq 2 \mu_{1}-\frac{\mu_{2}}{\sqrt{1-d}} .
$$

Note that, from (15), such a constant $\xi$ exists.

Lemma 2.2. Let $\Phi=(u, v, \varphi, \psi, z)^{T}$, then

$$
\|\Phi\|_{t} \leq\|\Phi\|_{s} e^{\frac{d}{2 \tau} 0 t-s \mid}, \forall t, s \in[0, T],
$$

where $d$ is a positive constant. 
Proof. For all $s, t \in[0, T]$, we have

$$
\begin{aligned}
& \|\Phi\|_{t}^{2}-\|\Phi\|_{s}^{2} e^{\left(\frac{d}{2 \bar{\tau}_{0}}\right)|t-s|} \\
& =\left(1-e^{\left(\frac{d}{2 \bar{\tau}_{0}}\right)|t-s|}\right)\left(\int_{\Omega}\left(\left\{\varphi^{2}+a u_{x}^{2}\right\}\right) d x+\int_{L_{1}}^{L_{2}}\left\{\psi^{2}+b v_{x}^{2}\right\} d x\right) \\
& +\xi\left(\tau(t)-\tau(s) e^{\left(\frac{d}{2 \bar{\tau}_{0}}\right)|t-s|}\right) \int_{\Omega} \int_{0}^{1} z^{2}(x, \rho) d \rho d x .
\end{aligned}
$$

We notice that

$$
e^{\left(\frac{d}{2 \bar{\tau}_{0}}\right)|t-s|} \geq 1
$$

Moreover

for some $d>0$.

$$
\tau(t)-\tau(s) e^{\left(\frac{d}{2 \tau_{0}}\right)|t-s|} \leq 0,
$$

Indeed,

$$
\tau(t)=\tau(s)+\tau^{\prime}(a)(t-s),
$$

where $a, b \in(s, t)$, and thus,

$$
\frac{\tau(t)}{\tau(s)}=1+\frac{\left|\tau^{\prime}(a)\right|}{\tau(s)}|t-s|,
$$

By (4), $\tau^{\prime}$ is bounded on $[0, T]$ and therefore, recalling also (2),

$$
\frac{\tau(t)}{\tau(s)} \leq 1+\frac{d}{\bar{\tau}_{0}}|t-s| \leq e^{\frac{d}{2 \tau_{0}}|t-s|}
$$

thus

This complete the proof.

$$
\frac{\tau(t)}{\tau(s)} \leq e^{\frac{d}{2 \tau}|t-s|} .
$$

Lemma 2.3. Under condition (16) the operator

$$
\mathcal{A}_{1}(t)=\mathcal{A}(t)-\kappa(t) I,
$$

is dissipative, and

$$
\frac{d}{d t} \mathcal{A}_{1}(t) \in L_{*}^{\infty}([0, T], B(D(A(0)), \mathcal{H})),
$$

where

$$
\kappa(t)=\frac{\sqrt{\tau^{\prime 2}(t)+1}}{2 \tau(t)} .
$$

Proof. Taking $U=(u, v, \varphi, \psi, z)^{T} \in D(\mathcal{A}(t))$. Then, for a fixed $t$,

$$
\left\langle\mathcal{A}(t)\left(\begin{array}{c}
u \\
v \\
\varphi \\
\psi \\
z
\end{array}\right),\left(\begin{array}{c}
u \\
v \\
\varphi \\
\psi \\
z
\end{array}\right)\right\rangle_{t}
$$


$=\left\langle\left(\begin{array}{c}\varphi \\ \psi \\ a u_{x x}-\mu_{1} \varphi-\mu_{2} z(., 1) \\ b v_{x x} \\ \frac{\tau^{\prime}(t) \rho-1}{\tau(t)} z_{\rho}\end{array}\right),\left(\begin{array}{c}u \\ v \\ \varphi \\ \psi \\ z\end{array}\right)\right\rangle_{t}$

$=\int_{\Omega}\left\{\left(a u_{x x}-\mu_{1} \varphi-\mu_{2} z(., 1)\right) \varphi+a \varphi_{x} u_{x}\right\} d x+\int_{L_{1}}^{L_{2}}\left\{b v_{x x} \psi+b \psi_{x} v_{x}\right\} d x$

$-\xi \tau(t) \int_{\Omega} \int_{0}^{1} \frac{1-\tau^{\prime}(t) \rho}{\tau(t)} z_{\rho} z(x, \rho) d \rho d x$.

$=a \int_{\Omega} \varphi u_{x x} d x-\mu_{1} \int_{\Omega} \varphi^{2} d x-\mu_{2} \int_{\Omega} \varphi z(., 1) d x+a \int_{\Omega} \varphi_{x} u_{x} d x$

$+b \int_{L_{1}}^{L_{2}} v_{x x} \psi d x+b \int_{L_{1}}^{L_{2}} \psi_{x} v_{x} d x-\xi \int_{\Omega} \int_{0}^{1}\left(1-\tau^{\prime}(t) \rho\right) z_{\rho} z(x, \rho) d \rho d x$.

Integrating by parts, we obtain

$$
\begin{aligned}
& \int_{\Omega} \int_{0}^{1}\left(1-\tau^{\prime}(t) \rho\right) z_{\rho} z(x, \rho) d \rho d x \\
& =\frac{\tau^{\prime}(t)}{2} \int_{\Omega} \int_{0}^{1} z^{2}(x, \rho) d \rho d x+\frac{1}{2} \int_{\Omega}\left\{z^{2}(x, 1)\left(1-\tau^{\prime}(t)\right)-z^{2}(x, 0)\right\} d x .
\end{aligned}
$$

We get

$$
\begin{aligned}
\langle\mathcal{A}(t) U, U\rangle_{t} & =a \int_{\Omega} \varphi u_{x x} d x-\mu_{1} \int_{\Omega} \varphi^{2} d x-\mu_{2} \int_{\Omega} \varphi z(., 1) d x+a \int_{\Omega} \varphi_{x} u_{x} d x \\
& +b \int_{L_{1}}^{L_{2}} v_{x x} \psi d x+b \int_{L_{1}}^{L_{2}} \psi_{x} v_{x} d x+\frac{\xi}{2} \int_{\Omega} z^{2}(x, 0) d x \\
& -\frac{\xi \tau^{\prime}(t)}{2} \int_{\Omega} \int_{0}^{1} z^{2}(x, \rho) d \rho d x-\frac{\xi\left(1-\tau^{\prime}(t)\right)}{2} \int_{\Omega} z^{2}(x, 1) d x,
\end{aligned}
$$

by fact that $z(x, 0)=\varphi(x)$

(20)

$$
\begin{aligned}
& \langle\mathcal{A}(t) U, U\rangle_{t} \\
& =a\left[u_{x} \varphi\right]_{\partial \Omega}+b\left[v_{x} \psi\right]_{L_{1}}^{L_{2}}-\mu_{1} \int_{\Omega} \varphi^{2} d x-\mu_{2} \int_{\Omega} \varphi z(., 1) d x \\
& +\frac{\xi}{2} \int_{\Omega} \varphi^{2} d x-\frac{\xi\left(1-\tau^{\prime}(t)\right)}{2} \int_{\Omega} z^{2}(x, 1) d x+\frac{\xi \tau^{\prime}(t)}{2} \int_{\Omega} \int_{0}^{1} z^{2}(x, \rho) d \rho,
\end{aligned}
$$

$$
\begin{aligned}
\langle\mathcal{A}(t) U, U\rangle_{t} & =a\left[u_{x} \varphi\right]_{\partial \Omega}+b\left[v_{x} \psi\right]_{L_{1}}^{L_{2}}-\left(\mu_{1}-\frac{\xi}{2}\right) \int_{\Omega} \varphi^{2} d x-\mu_{2} \int_{\Omega} \varphi z(., 1) d x \\
& -\frac{\xi\left(1-\tau^{\prime}(t)\right)}{2} \int_{\Omega} z^{2}(x, 1) d x+\frac{\xi \tau^{\prime}(t)}{2} \int_{\Omega} \int_{0}^{1} z^{2}(x, \rho) d \rho,
\end{aligned}
$$


Using Young's inequality, the third condition of (1) and the equality $\varphi\left(L_{2}\right)=$ $\psi\left(L_{2}\right)$, we obtain

$$
\begin{aligned}
\langle\mathcal{A}(t) U, U\rangle_{t} & \leq-\left(\mu_{1}-\frac{\xi}{2}-\frac{\mu_{2}}{2 \sqrt{1-d}}\right) \int_{\Omega} \varphi^{2} d x \\
& -\left(\frac{\xi(1-d)}{2}-\frac{\mu_{2} \sqrt{1-d}}{2}\right) \int_{\Omega} z^{2}(x, 1) d x+k(t)\langle U, U\rangle,
\end{aligned}
$$

where

$$
\kappa(t)=\frac{\left(\tau^{\prime}(t)^{2}+1\right)^{\frac{1}{2}}}{2 \tau(t)} .
$$

Consequently, using (16), we deduce that

$$
\langle\mathcal{A}(t) U, U\rangle_{t}-\kappa(t)\langle U, U\rangle_{t} \leq 0 \text {. }
$$

Which means that the operator

$$
\mathcal{A}(t)_{1}(t)=\mathcal{A}(t)-k(t) I
$$

is dissipative.

Moreover,

$$
\kappa^{\prime}(t)=\frac{\tau^{\prime \prime}(t) \tau^{\prime}(t)}{2 \tau(t)\left(\tau^{\prime 2}+1\right)^{\frac{1}{2}}}-\frac{\tau^{\prime}(t)\left(\tau^{\prime 2}(t)+1\right)^{\frac{1}{2}}}{2 \tau(t)^{2}},
$$

is bounded on $[0, T]$ for all $T>0$ (by (2) and (3) and we have

$$
\frac{d}{d t} \mathcal{A}(t) U=\left(0,0,0,0, \frac{\tau^{\prime \prime}(t) \tau(t) \rho-\tau^{\prime}(t)\left(\tau^{\prime}(t) \rho-1\right)}{\tau(t)^{2}} z_{\rho}\right)^{T}
$$

with

$$
\frac{\tau^{\prime \prime}(t) \tau(t) \rho-\tau^{\prime}(t)\left(\tau^{\prime}(t) \rho-1\right)}{\tau(t)^{2}}
$$

is bounded on $[0, T]$. Thus

$$
\frac{d}{d t} \mathcal{A}_{1}(t) \in L_{*}^{\infty}([0, T], B(D(\mathcal{A}(0)), \mathcal{H})),
$$

the space of equivalence classes of essentially bounded, strongly measurable functions from $[0, T]$ into $B(D(\mathcal{A}(0)), \mathcal{H})$.

Lemma 2.4. For fixed $t>0$ and $\lambda>0$, the operator $\lambda I-\mathcal{A}(t)$ is surjective. Proof. Let $\left(f, g, g_{1}, h_{1}, h_{2}\right)^{T} \in \mathcal{H}$, we seek $U=(u, v, \varphi, \psi, z)^{T} \in D(\mathcal{A}(t))$ solution of

$$
(\lambda I-\mathcal{A}(t))\left(\begin{array}{c}
u \\
v \\
\varphi \\
\psi \\
y
\end{array}\right)=\left(\begin{array}{c}
f \\
g \\
g_{1} \\
h_{1} \\
h_{2}
\end{array}\right),
$$


that is verifying

$$
\begin{aligned}
\lambda u-\varphi & =f, \\
\lambda v-\psi & =g, \\
\lambda \varphi-a u_{x x}+\mu_{1} \varphi+\mu_{2} z(., 1) & =g_{1}, \\
\lambda \psi-b v_{x x} & =h_{1}, \\
\lambda z-\frac{\tau^{\prime}(t) \rho-1}{\tau(t)} z_{\rho} & =h_{2} .
\end{aligned}
$$

Suppose that we have found $(u, v)$ with the appropriate regularity. Then

$$
\begin{aligned}
& \varphi=\lambda u-f, \\
& \psi=\lambda v-g .
\end{aligned}
$$

It is clear that $\varphi \in H^{1}(\Omega)$ and $\psi \in H^{1}\left(L_{1}, L_{2}\right)$, furthermore, by (25), we can find $z$ as $z(x, 0)=\varphi(x), x \in \Omega$, using the approach as in Nicaise and Pignotti [14], we obtain, by using the equation in (25)

$$
z(x, \rho)=\varphi(x) e^{-\lambda \rho \tau(t)}+\tau(t) e^{-\lambda \rho \tau(t)} \int_{0}^{\rho} h_{2}(x, \sigma) e^{\lambda \sigma \tau(t)} d \sigma,
$$

if $\tau^{\prime}(t)=0$, and

$$
\begin{aligned}
z(x, \rho) & =\varphi(x) e^{\lambda \frac{\tau(t)}{\tau^{\prime}(t)} \ln \left(1-\tau^{\prime}(t) \rho\right)} \\
& +e^{\lambda \frac{\tau(t)}{\tau^{\prime}(t)} \ln \left(1-\tau^{\prime}(t) \rho\right)} \int_{0}^{\rho} \frac{h_{2}(x, \sigma) \tau(t)}{1-\tau^{\prime}(t) \sigma} e^{-\lambda \frac{\tau(t)}{\tau^{\prime}(t)} \ln \left(1-\tau^{\prime}(t) \sigma\right)} d \sigma
\end{aligned}
$$

otherwise.

By using (25), (26) and (27), the functions $u, v$ satisfy the following equations

$$
\begin{aligned}
\lambda^{2} u-a u_{x x}+\mu_{1} z(., 0)+\mu_{2} z(., 1) & =g_{1}+\lambda f, \\
\lambda^{2} v-b v_{x x} & =h_{1}+\lambda g .
\end{aligned}
$$

Since

$$
\begin{aligned}
z(x, 1) & =\lambda u e^{-\lambda \tau(t)}-f e^{-\lambda \tau(t)}+\tau(t) e^{-\lambda \tau(t)} \int_{0}^{1} h_{2}(x, \sigma) e^{\lambda \sigma \tau(t)} d \sigma \\
& =\lambda u e^{-\lambda \tau(t)}+z_{0}(x)
\end{aligned}
$$

with

$$
z_{0}(x)=-f e^{-\lambda \tau(t)}+\tau(t) e^{-\lambda \tau(t)} \int_{0}^{1} h_{2}(x, \sigma) e^{\lambda \sigma \tau(t)} d \sigma, \text { for } x \in \Omega,
$$

if $\tau^{\prime}(t)=0$, and

$$
\begin{aligned}
z(x, 1) & =\lambda u e^{\lambda \frac{\tau(t)}{\tau^{\prime}(t)} \ln \left(1-\tau^{\prime}(t)\right)}-f e^{\lambda \frac{\tau(t)}{\tau^{\prime}(t)} \ln \left(1-\tau^{\prime}(t)\right)} \\
& +e^{\lambda \frac{\tau(t)}{\tau^{\prime}(t)} \ln \left(1-\tau^{\prime}(t)\right)} \int_{0}^{1} \frac{h_{2}(x, \sigma) \tau(t)}{1-\tau^{\prime}(t) \sigma} e^{-\lambda \frac{\tau(t)}{\tau^{\prime}(t)} \ln \left(1-\tau^{\prime}(t) \sigma\right)} d \sigma
\end{aligned}
$$




$$
=\lambda u e^{\lambda \frac{\tau(t)}{\tau^{\prime}(t)} \ln \left(1-\tau^{\prime}(t)\right)}+z_{0}(x), \text { for } x \in \Omega,
$$

with

$$
\begin{aligned}
z_{0}(x) & =-f e^{\lambda \frac{\tau(t)}{\tau^{\prime}(t)} \ln \left(1-\tau^{\prime}(t)\right)} \\
& +e^{\lambda \frac{\tau(t)}{\tau^{\prime}(t)} \ln \left(1-\tau^{\prime}(t)\right)} \int_{0}^{1} \frac{h_{2}(x, \sigma) \tau(t)}{1-\tau^{\prime}(t) \sigma} e^{-\lambda \frac{\tau(t)}{\tau^{\prime}(t)} \ln \left(1-\tau^{\prime}(t) \sigma\right)} d \sigma
\end{aligned}
$$

otherwise.

The system (25)-(26) can be reformulated as

$$
\left\{\begin{array}{r}
\int_{\Omega}\left(\lambda^{2} u-a u_{x x}+\mu_{1} \lambda u+\lambda \mu_{2} u e^{-\lambda \tau(t)}\right) \omega d x \\
=\int_{\Omega}\left(\mu_{1} f+g_{1}+\lambda f-\mu_{2} z_{0}(x)\right) \omega d x \\
\int_{L_{1}}^{L_{2}}\left(\lambda^{2} v-b v_{x x}\right) \tilde{\omega} d x=\int_{L_{1}}^{L_{2}}\left(h_{1}+\lambda g\right) \tilde{\omega} d x
\end{array}\right.
$$

for any $(\omega, \tilde{\omega}) \in X_{*}$, if $\tau^{\prime}(t)=0$, and

$$
\left\{\begin{array}{c}
\int_{\Omega}\left(\lambda^{2} u-a u_{x x}+\mu_{1} \lambda u+\lambda \mu_{2} u e^{\lambda \frac{\tau(t)}{\tau^{\prime}(t)} \ln \left(1-\tau^{\prime}(t)\right)}\right) \omega d x \\
=\int_{\Omega}\left(\mu_{1} f+g_{1}+\lambda f-\mu_{2} z_{0}(x)\right) \omega d x, \\
\int_{L_{1}}^{L_{2}}\left(\lambda^{2} v-b v_{x x}\right) \tilde{\omega} d x=\int_{L_{1}}^{L_{2}}\left(h_{1}+\lambda g\right) \tilde{\omega} d x,
\end{array}\right.
$$

otherwise.

Integrating by parts, we obtain

$$
\left\{\begin{array}{c}
\int_{\Omega}\left(\lambda^{2}+\mu_{1} \lambda+\lambda \mu_{2} e^{-\lambda \tau(t)}\right) u \omega d x+a \int_{\Omega} u_{x} \omega_{x} d x-a\left[u_{x} \omega\right]_{\partial \Omega} \\
=\int_{\Omega}\left(\mu_{1} f+g_{1}+\lambda f-\mu_{2} z_{0}(x)\right) \omega d x \\
\int_{L_{1}}^{L_{2}} \lambda^{2} v \tilde{\omega} d x+b \int_{L_{1}}^{L_{2}} v_{x} \tilde{\omega}_{x} d x-b\left[v_{x} \tilde{\omega}\right]_{L_{1}}^{L_{2}}=\int_{L_{1}}^{L_{2}}\left(h_{1}+\lambda g\right) \tilde{\omega} d x
\end{array}\right.
$$

if $\tau^{\prime}(t)=0$, and

$$
\left\{\begin{array}{c}
\int_{\Omega}\left(\lambda^{2}+\mu_{1} \lambda+\lambda \mu_{2} e^{\lambda \frac{\tau(t)}{\tau^{\prime}(t)} \ln \left(1-\tau^{\prime}(t)\right)}\right) u \omega d x+a \int_{\Omega} u_{x} \omega_{x} d x-a\left[u_{x} \omega\right]_{\partial \Omega} \\
=\int_{\Omega}\left(\mu_{1} f+g_{1}+\lambda f-\mu_{2} z_{0}(x)\right) \omega d x, \\
\int_{L_{1}}^{L_{2}} \lambda^{2} v \tilde{\omega} d x+b \int_{L_{1}}^{L_{2}} v_{x} \tilde{\omega}_{x} d x-b\left[v_{x} \tilde{\omega}\right]_{L_{1}}^{L_{2}}=\int_{L_{1}}^{L_{2}}\left(h_{1}+\lambda g\right) \tilde{\omega} d x,
\end{array}\right.
$$

otherwise. The problem (32) and (33) is equivalent to the problem,

$$
\Phi((u, v),(\omega, \tilde{\omega}))=l(\omega, \tilde{\omega}),
$$

where the bilinear form $\Phi:\left(X_{*} \times X_{*}\right) \rightarrow \mathbb{R}$ and the linear form $l: X_{*} \rightarrow \mathbb{R}$ are defined by

$$
\begin{aligned}
& \Phi((u, v),(\omega, \tilde{\omega})) \\
& =\int_{\Omega}\left(\lambda^{2}+\mu_{1} \lambda+\lambda \mu_{2} e^{-\lambda \tau(t)}\right) u \omega d x+a \int_{\Omega} u_{x} \omega_{x} d x-a\left[u_{x} \omega\right]_{\partial \Omega} \\
& +\int_{L_{1}}^{L_{2}} \lambda^{2} v \tilde{\omega} d x+b \int_{L_{1}}^{L_{2}} v_{x} \tilde{\omega}_{x} d x-b\left[v_{x} \tilde{\omega}\right]_{L_{1}}^{L_{2}},
\end{aligned}
$$




$$
l(\omega, \tilde{\omega})=\int_{\Omega}\left(\mu_{1} f+g_{1}+\lambda f-\mu_{2} y_{0}(x)\right) \omega d x+\int_{L_{1}}^{L_{2}}\left(h_{1}+\lambda g\right) \tilde{\omega} d x,
$$

if $\tau^{\prime}(t)=0$, and

$$
\begin{aligned}
& \Phi((u, v),(\omega, \tilde{\omega})) \\
& =\int_{\Omega}\left(\lambda^{2}+\mu_{1} \lambda+\lambda \mu_{2} e^{\lambda \frac{\tau(t)}{\tau^{\prime}(t)} \ln \left(1-\tau^{\prime}(t)\right)}\right) u \omega d x+a \int_{\Omega} u_{x} \omega_{x} d x-a\left[u_{x} \omega\right]_{\partial \Omega} \\
& +\int_{L_{1}}^{L_{2}} \lambda^{2} v \tilde{\omega} d x+b \int_{L_{1}}^{L_{2}} v_{x} \tilde{\omega}_{x} d x-b\left[v_{x} \tilde{\omega}\right]_{L_{1}}^{L_{2}}, \\
& \quad l(\omega, \tilde{\omega})=\int_{\Omega}\left(\mu_{1} f+g_{1}+\lambda f-\mu_{2} z_{0}(x)\right) \omega d x+\int_{L_{1}}^{L_{2}}\left(h_{1}+\lambda g\right) \tilde{\omega} d x,
\end{aligned}
$$

otherwise.

Using the properties of the space $X_{*}$, it is clear that $\Phi$ is continuous and coercive, and $l$ is continuous. So applying the Lax-Milgram theorem, we deduce that for all $(\omega, \tilde{\omega}) \in X_{*}$, problem (34) admits a unique solution $(u, v) \in$ $X_{*}$. It follows from (32) and (33) that $(u, v) \in\left\{\left(H^{2}(\Omega) \times H^{2}\left(L_{1}, L_{2}\right)\right) \cap X_{*}\right\}$. In conclusion, we have found $U=(u, v, \varphi, \psi, z)^{T} \in D(\mathcal{A}(t))$, which verifies (25), and thus $(\lambda I-\mathcal{A}(t))$ is surjective for some $\lambda>0$ and $t>0$. Again as $\kappa(t)>0$, this proves that

$$
\lambda I-\mathcal{A}_{1}(t)=(\lambda+\kappa(t)) I-\mathcal{A}(t), \quad \text { is surjective, }
$$

for any $\lambda>0$ and $t>0$.

Theorem 2.2. The operator $A$ generates a $C_{0}$-semigroup on $H$. For any $U_{0} \in \mathcal{H}$, the problem (9) possesses a unique weak solution $U \in C([0,+\infty), \mathcal{H})$. Moreover, if $U_{0} \in D(\mathcal{A}(0))$, then $U$ is a strong solution, i.e

$$
U \in C([0,+\infty), D(\mathcal{A}(0))) \cap C^{1}([0,+\infty), \mathcal{H}) .
$$

Proof. Results (17), (18) and (35) imply that the family $\mathcal{A}_{1}=\left\{\mathcal{A}_{1}(t)\right.$ : $t \in[0, T]\}$ is a stable family of generators in $\mathcal{H}$ with stability constants independent of $t$. Therefore, all assumptions of Theorem 2.1 are satisfied by (13), Lemma2.1-Lemma2.4, and thus, the problem

$$
\begin{aligned}
\tilde{U}^{\prime} & =\mathcal{A}_{1}(t) \tilde{U}, \\
\tilde{U}(0) & =U_{0},
\end{aligned}
$$

has a unique solution $\tilde{U} \in C([0,+\infty), D(\mathcal{A}(0))) \cap C^{1}([0,+\infty), \mathcal{H})$ for $U_{0} \in$ $D(\mathcal{A}(0))$. The requested solution of $(2)$ is then given by

$$
U(t)=e^{B(t)} \tilde{U}(t),
$$

with $B(t)=\int_{0}^{t} \kappa(s) d s$ because

$$
\begin{aligned}
U^{\prime}(t) & =\kappa(t) e^{B(t)} \tilde{U}(t)+e^{B(t)} \tilde{U}^{\prime}(t), \\
U^{\prime}(t) & =\kappa(t) e^{B(t)} \tilde{U}(t)+e^{B(t)} \mathcal{A}_{1}(t) \tilde{U}(t),
\end{aligned}
$$




$$
U^{\prime}(t)=\mathcal{A}(t) e^{B(t)} \tilde{U}(t)=\mathcal{A}(t) U(t) .
$$

This concludes the proof.

\section{Stability REsult}

In this section we study the asymptotic behavior of the system (1). For any regular solution of (1), we give the total energy as

$$
\begin{aligned}
E(t) & =\frac{1}{2} \int_{\Omega} u_{t}^{2}(x, t) d x+\frac{a}{2} \int_{\Omega} u_{x}^{2}(x, t) d x+\frac{1}{2} \int_{L_{1}}^{L_{2}} v_{t}^{2}(x, t) d x \\
& +\frac{b}{2} \int_{L_{1}}^{L_{2}} v_{x}^{2}(x, t) d x+\frac{\xi}{2} \int_{\Omega} \int_{t-\tau(t)}^{t} u_{t}^{2}(x, s) d s d x,
\end{aligned}
$$

where $\xi$ is the positive constant defined by (16). Our next main result reads as.

Theorem 3.1. Let $(u, v)$ be the solution of (1). Assume that $\mu_{2}>\mu_{1}$ and

$$
\frac{a}{b}<\frac{L_{3}+L_{1}-L_{2}}{2\left(L_{2}-L_{1}\right)} \text {. }
$$

Then there exist two positive constants $W$ and $w$, such that

$$
E(t) \leq W e^{-w t}, \quad \forall t \geq 0 .
$$

To prove Theorem 3.1, we use the following lemmas. First, we will need an explicit formula of energy derivative. The following energy functional law holds.

Lemma 3.1. Let $(u, v, z)$ be the solution of (1). Assume that $\mu_{1} \geq \mu_{2}$. Then we have the inequality

$$
\begin{aligned}
\frac{d E(t)}{d t} \leq & \left(-\mu_{1}+\frac{\mu_{2} \sqrt{1-d}}{2}+\frac{\xi}{2}\right) \int_{\Omega} u_{t}^{2}(x, t) d x \\
& -\left(\frac{\xi(1-d)}{2}-\frac{\mu_{2}}{2 \sqrt{1-d}}\right) \int_{\Omega} u_{t}^{2}(x, t-\tau(t)) d x .
\end{aligned}
$$

Proof. From (36) we have

$$
\begin{aligned}
\frac{d E_{1}(t)}{d t} & =a \int_{\Omega} u_{t}(x, t) u_{x x}(x, t) d x-\mu_{1} \int_{\Omega} u_{t}^{2}(x, t) d x \\
& -\mu_{2} \int_{\Omega} u_{t}(x, t) u_{t}(x, t-\tau(t)) d x+a \int_{\Omega} u_{x t}(x, t) u_{x}(x, t) d x .
\end{aligned}
$$

where

$$
E_{1}(t)=\frac{1}{2} \int_{\Omega} u_{t}^{2}(x, t) d x+\frac{a}{2} \int_{\Omega} u_{x}^{2}(x, t) d x .
$$

Using system (2), and integrating by parts, we obtain

$$
\frac{d E_{1}(t)}{d t}=a \int_{\Omega} u_{t}(x, t) u_{x x}(x, t) d x-\mu_{1} \int_{\Omega} u_{t}^{2}(x, t) d x
$$




$$
\begin{aligned}
& -\mu_{2} \int_{\Omega} u_{t}(x, t) u_{t}(x, t-\tau(t)) d x \\
& +a\left[u_{x} u_{t}\right]_{\partial \Omega}-a \int_{\Omega} u_{x x}(x, t) u_{t}(x, t) d x .
\end{aligned}
$$

applying Young's inequality

$$
\begin{aligned}
\frac{d E_{1}(t)}{d t} \leq & -\left(\mu_{1}-\frac{\mu_{2} \sqrt{1-d}}{2}\right) \int_{\Omega} u_{t}^{2}(x, t) d x \\
& +\frac{\mu_{2}}{2 \sqrt{1-d}} \int_{\Omega} u_{t}^{2}(x, t-\tau(t)) d x+a\left[u_{x} u_{t}\right]_{\partial \Omega} .
\end{aligned}
$$

On the other hand,

$$
\frac{d E_{2}(t)}{d t}=b\left[v_{x} v_{t}\right]_{L_{1}}^{L_{2}} .
$$

where

$$
E_{2}(t)=\frac{1}{2} \int_{L_{1}}^{L_{2}} v_{t}^{2}(x, t) d x+\frac{b}{2} \int_{L_{1}}^{L_{2}} v_{x}^{2}(x, t) d x .
$$

Using the fact that

$$
\begin{aligned}
& \frac{d}{d t} \int_{\Omega} \int_{t-\tau(t)}^{t} u_{t}^{2}(x, s) d s d x \\
& =\int_{\Omega} u_{t}^{2}(x, t) d x-\left(1-\tau^{\prime}(t)\right) \int_{\Omega} u_{t}^{2}(x, t-\tau(t)) d x,
\end{aligned}
$$

collecting (40), (41), (42), using boundary conditions and applying Young's inequality, we show that (39) holds. The proof is complete.

Following [2], we define the functional

$$
I(t)=\int_{\Omega} \int_{t-\tau(t)}^{t} e^{s-t} u_{t}^{2}(x, s) d s d x,
$$

and state the following lemma.

Lemma 3.2. Let $(u, v)$ be the solution of (2). Then

$$
\begin{aligned}
\frac{d I(t)}{d t} \leq & \int_{\Omega} u_{t}^{2}(x, t) d x-(1-d) e^{-\bar{\tau}} \int_{\Omega} u_{t}^{2}(x, t-\tau(t)) d x \\
& -e^{-\bar{\tau}} \int_{\Omega} \int_{t-\tau(t)}^{t} u_{t}^{2}(x, s) d s d x .
\end{aligned}
$$

Now, we define the functional $\mathfrak{D}(t)$ as follows

$$
\mathfrak{D}(t)=\int_{\Omega} u u_{t} d x+\frac{\mu_{1}}{2} \int_{\Omega} u^{2} d x+\int_{L_{1}}^{L_{2}} v v_{t} d x .
$$

Then, we have the following estimate. 
Lemma 3.3. The functional $\mathfrak{D}(t)$ satisfies

$$
\begin{aligned}
\frac{d \mathfrak{D}(t)}{d t} & \leq-\left(a-\epsilon_{0} c_{0}^{2}\right) \int_{\Omega} u_{x}^{2}(x, t) d x-b \int_{L_{1}}^{L_{2}} v_{x}^{2}(x, t) d x \\
& +\int_{\Omega} u_{t}^{2} d x+\int_{L_{1}}^{L_{2}} v_{t}^{2} d x+C\left(\epsilon_{0}\right) \int_{\Omega} u_{t}^{2}(x, t-\tau(t)) d x .
\end{aligned}
$$

Proof. Taking the derivative of $\mathfrak{D}(t)$ with respect to $t$ and using (1), we find that

$$
\begin{aligned}
\frac{d \mathfrak{D}(t)}{d t} & =\int_{\Omega} u_{t}^{2} d x+\int_{L_{1}}^{L_{2}} v_{t}^{2} d x-a \int_{\Omega} u_{x}^{2}(x, t) d x-b \int_{L_{1}}^{L_{2}} v_{x}^{2}(x, t) d x \\
& -\mu_{2} \int_{\Omega} u u_{t}(x, t-\tau(t)) d x+a\left[u u_{x}\right]_{\partial \Omega}+b\left[v v_{x}\right]_{L_{1}}^{L_{2}} .
\end{aligned}
$$

Using the boundary conditions, we have

$$
a\left[u u_{x}\right]_{\partial \Omega}+b\left[v v_{x}\right]_{L_{1}}^{L_{2}}=0 .
$$

On the other hand, we have by Poincaré's and Young's inequalities,

$$
\begin{aligned}
\mu_{2} \int_{\Omega} u u_{t}(x, t-\tau(t)) d x & \leq \epsilon_{0} \int_{\Omega} u^{2} d x+C\left(\epsilon_{0}\right) \int_{\Omega} u_{t}^{2}(x, t-\tau(t)) d x \\
& \leq \epsilon_{0} c_{0}^{2} \int_{\Omega} u_{x}^{2} d x+C\left(\epsilon_{0}\right) \int_{\Omega} u_{t}^{2}(x, t-\tau(t)) d x,
\end{aligned}
$$

where $c_{0}$ is the Poincaé's constant. Consequently, plugging the above estimates into (46), we find (45).

Now, inspired by [10], we introduce the functional

$$
q(x)= \begin{cases}x-\frac{L_{1}}{2}, & x \in\left[0, L_{1}\right], \\ x-\frac{L_{2}+L_{3}}{2}, & x \in\left[L_{2}, L_{3}\right], \\ \frac{L_{2}-L_{3}-L_{1}}{2\left(L_{2}-L_{1}\right)}\left(x-L_{1}\right)+\frac{L_{1}}{2}, & x \in\left[L_{1}, L_{2}\right] .\end{cases}
$$

Next, in order to construct the Lyapounov function, we define the functionals

$$
\mathcal{L}_{1}(t)=-\int_{\Omega} q(x) u_{x} u_{t} d x, \quad \mathcal{L}_{2}(t)=-\int_{L_{1}}^{L_{2}} q(x) v_{x} v_{t} d x .
$$

Then, we have the following estimates.

Lemma 3.4. For any $\epsilon_{2}>0$, we have the estimates

$$
\begin{aligned}
\frac{d \mathcal{L}_{1}(t)}{d t} & \leq C\left(\epsilon_{2}\right) \int_{\Omega} u_{t}^{2} d x+\left(\frac{a}{2}+\epsilon_{2}\right) \int_{\Omega} u_{x}^{2} d x \\
& +C\left(\epsilon_{2}\right) \int_{\Omega} u_{t}^{2}(x, t-\tau(t)) d x-\frac{a}{4}\left[\left(L_{3}-L_{2}\right) u_{x}^{2}\left(L_{3}, t\right)+L_{1} u_{x}^{2}\left(L_{2}, t\right)\right],
\end{aligned}
$$


and

$$
\begin{aligned}
\frac{d \mathcal{L}_{2}(t)}{d t} \leq & \frac{L_{2}-L_{3}-L_{1}}{4\left(L_{2}-L_{1}\right)}\left(\int_{L_{1}}^{L_{2}} v_{t}^{2} d x+\int_{L_{1}}^{L_{2}} b v_{x}^{2} d x\right) \\
& +\frac{b}{4}\left(\left(L_{3}-L_{2}\right) v_{x}^{2}\left(L_{2}, t\right)+L_{1} v_{x}^{2}\left(L_{1}, t\right)\right) .
\end{aligned}
$$

Proof. Taking the derivative of $\mathcal{L}_{1}(t)$ with respect to $t$ and using (1), we obtain

$$
\begin{aligned}
\frac{d \mathcal{L}_{1}(t)}{d t} & =-\int_{\Omega} q(x) u_{x t} u_{t} d x-a \int_{\Omega} q(x) u_{x} u_{x x}(x, t) d x \\
& +\mu_{1} \int_{\Omega} q(x) u_{x} u_{t}(x, t) d x+\mu_{2} \int_{\Omega} q(x) u_{x} u_{t}(x, t-\tau(t)) d x
\end{aligned}
$$

Integrating by parts,

$$
\int_{\Omega} q(x) u_{t x} u_{t} d x=-\frac{1}{2} \int_{\Omega} q^{\prime}(x) u_{t}^{2} d x+\frac{1}{2}\left[q(x) u_{t}^{2}\right]_{\partial \Omega} .
$$

On the other hand,

$$
\int_{\Omega} a q(x) u_{x x} u_{x} d x=-\frac{1}{2} \int_{\Omega} a q^{\prime}(x) u_{x}^{2} d x+\frac{1}{2}\left[a q(x) u_{x}^{2}\right]_{\partial \Omega} .
$$

Substituting (52) and (53) in (51), we find that

$$
\begin{aligned}
\frac{d \mathcal{L}_{1}(t)}{d t} & =\frac{1}{2} \int_{\Omega} q^{\prime}(x) u_{t}^{2} d x-\frac{1}{2}\left[q(x) u_{t}^{2}\right]_{\partial \Omega}+\frac{1}{2} \int_{\Omega} a q^{\prime}(x) u_{x}^{2} d x-\frac{1}{2}\left[a q(x) u_{x}^{2}\right]_{\partial \Omega} \\
& +\mu_{1} \int_{\Omega} q(x) u_{x} u_{t}(x, t) d x+\mu_{2} \int_{\Omega} q(x) u_{x} u_{t}(x, t-\tau(t)) d x .
\end{aligned}
$$

Using Young's inequality and (48), equation (54) becomes

$$
\begin{aligned}
\frac{d \mathcal{L}_{1}(t)}{d t} & \leq C\left(\epsilon_{2}\right) \int_{\Omega} u_{t}^{2} d x+\left(\frac{a}{2}+\epsilon_{2}\right) \int_{\Omega} u_{x}^{2} d x \\
& +C\left(\epsilon_{2}\right) \int_{\Omega} u_{t}^{2}(x, t-\tau(t)) d x-\frac{a}{2}\left[q(x) u_{x}^{2}\right]_{\partial \Omega}-\frac{1}{2}\left[q(x) u_{t}^{2}\right]_{\partial \Omega}
\end{aligned}
$$

Since $q\left(L_{1}\right)>0$ and $q\left(L_{2}\right)<0$, by using the boundary condition, we have

$$
\frac{1}{2}\left[q(x) u_{t}^{2}\right]_{\partial \Omega} \geq 0 \text {. }
$$

Also, we have

$$
-\frac{a}{2}\left[q(x) u_{x}^{2}\right]_{\partial \Omega}=-\frac{a\left(L_{3}-L_{2}\right)}{4}\left[u_{x}^{2}\left(L_{3}, t\right)+u_{x}^{2}\left(L_{2}, t\right)\right] .
$$

Taking into account (56) and (57), then (55) gives (49). 
By the same method, taking the derivative of $\mathcal{L}_{2}(t)$ with respect to $t$, we obtain

$$
\begin{aligned}
\frac{d \mathcal{L}_{2}(t)}{d t} & =-\int_{L_{1}}^{L_{2}} q(x) v_{x t} v_{t} d x-\int_{L_{1}}^{L_{2}} q(x) v_{x} v_{t t} d x \\
& =\frac{1}{2} \int_{L_{1}}^{L_{2}} q^{\prime}(x) v_{t}^{2} d x+\frac{1}{2} \int_{L_{1}}^{L_{2}} b q^{\prime}(x) v_{x}^{2} d x-\frac{1}{2}\left[q(x) v_{t}^{2}\right]_{L_{1}}^{L_{2}}-\frac{b}{2}\left[q(x) u_{x}^{2}\right]_{L_{1}}^{L_{2}} \\
& \leq \frac{L_{2}-L_{3}-L_{1}}{4\left(L_{2}-L_{1}\right)}\left(\int_{L_{1}}^{L_{2}} v_{t}^{2} d x+\int_{L_{1}}^{L_{2}} b v_{x}^{2} d x\right) \\
& +\frac{b}{4}\left(\left(L_{3}-L_{2}\right) v_{x}^{2}\left(L_{2}, t\right)+L_{1} v_{x}^{2}\left(L_{1}, t\right)\right)
\end{aligned}
$$

which is exactly (50).

We define the Lyapunov functional

$$
\mathfrak{L}(t)=N E(t)+I(t)+\gamma_{2} \mathfrak{D}(t)+\gamma_{3} \mathcal{L}_{1}(t)+\gamma_{4} \mathcal{L}_{2}(t),
$$

where $N, \gamma_{2}, \gamma_{3}$ and $\gamma_{4}$ are positive constants.

Proof of the Theorem 3.1. Now, it is clear from the boundary conditions, that

$$
a^{2} u_{x}^{2}\left(L_{i}, t\right)=b^{2} v_{x}^{2}\left(L_{i}, t\right), \quad i=1,2 .
$$

Taking the derivative of (59) with respect to $t$ and making use of (39)-(49) and taking into account (60), we obtain

$$
\begin{aligned}
\frac{d \mathfrak{L}(t) \leq}{d t} \leq & \left\{N\left(-\mu_{1}+\frac{\mu_{2} \sqrt{1-d}}{2}+\frac{\xi}{2}\right)+1+\gamma_{2}+\gamma_{3} C\left(\epsilon_{2}\right)\right\} \int_{\Omega} u_{t}^{2}(x, t) d x \\
(61) & +\left\{N\left(\frac{\mu_{2}}{2 \sqrt{1-d}}-\frac{\xi(1-d)}{2}\right)-(1-d) e^{-\bar{\tau}}\right. \\
& \left.+\gamma_{2} C\left(\epsilon_{0}\right)+\gamma_{3} C\left(\epsilon_{2}\right)\right\} \int_{\Omega} u_{t}^{2}(x, t-\tau(t)) d x \\
& +\left(\gamma_{2}\left(-a+\epsilon_{0} c_{0}^{2}\right)+\gamma_{3} \epsilon_{2}+\frac{\gamma_{3} a}{2}\right) \int_{\Omega} u_{x}^{2}(x, t) d x \\
& +\left(\gamma_{4} \frac{L_{2}-L_{3}-L_{1}}{4\left(L_{2}-L_{1}\right)}-\gamma_{2} b\right) \int_{L_{1}}^{L_{2}} v_{x}^{2}(x, t) d x \\
& +\left(\gamma_{2}+\gamma_{4} \frac{L_{2}-L_{3}-L_{1}}{4\left(L_{2}-L_{1}\right)}\right) \int_{L_{1}}^{L_{2}} v_{t}^{2} d x \\
& -e^{-\bar{\tau}} \int_{\Omega}^{t} \int_{t-\tau(t)}^{t} u_{t}^{2}(x, s) d s d x-\left(\gamma_{3}-\frac{a}{b} \gamma_{4}\right) \frac{a\left(L_{3}-L_{2}\right)}{4} u_{x}^{2}\left(L_{2}, t\right) \\
& -\left(\gamma_{3}-\frac{a}{b} \gamma_{4}\right) \frac{a L_{1}}{4} u_{x}^{2}\left(L_{1}, t\right) .
\end{aligned}
$$


At this point, we choose our constants in (61), carefully, such that all the coefficients in (61) will be negative. Indeed, under the assumption (37), we can always find $\gamma_{2}, \gamma_{3}$ and $\gamma_{4}$ such that

$$
\frac{L_{2}-L_{3}-L_{1}}{4\left(L_{2}-L_{1}\right)} \gamma_{4}+\gamma_{2}<0, \quad \gamma_{3}>\frac{a}{b} \gamma_{4}, \quad \gamma_{2}>\frac{\gamma_{3}}{2} .
$$

Once the above constants are fixed, we may choose $\epsilon_{2}$ and $\epsilon_{0}$ small enough such that

$$
\gamma_{2} \epsilon_{0} c_{0}^{2}+\gamma_{3} \epsilon_{2}<a\left(\gamma_{2}-\frac{\gamma_{3}}{2}\right)
$$

Finally, keeping in mind (2) and choosing $N$ large enough such that the first and the second coefficients in (61) are negatives.

Consequently, from the above, we deduce that there exist a positive constant $\eta_{1}$, such that $(61)$ becomes

$$
\begin{aligned}
\frac{d \mathfrak{L}(t)}{d t} & \leq-\eta_{1} \int_{\Omega}\left(u_{t}^{2}(x, t)+u_{x}^{2}(x, t)+u_{t}^{2}(x, t-\tau(t))\right) d x \\
& -\eta_{1} \int_{\Omega}\left(v_{t}^{2}(x, t)+v_{x}^{2}(x, t)\right) d x-\eta_{1} \int_{\Omega} \int_{t-\tau(t)}^{t} u_{t}^{2}(x, s) d s d x
\end{aligned}
$$

Consequently, recalling (36), we deduce that there exist also $\eta_{2}>0$, such that

$$
\frac{d \mathfrak{L}(t)}{d t} \leq-\eta_{2} E(t), \quad \forall t \geq 0
$$

On the other hand, it is not hard to see that from (59) and for $N$ large enough, there exist two positive constants $\beta_{1}$ and $\beta_{2}$ such that

$$
\beta_{1} E(t) \leq \mathfrak{L}(t) \leq \beta_{2} E(t), \quad \forall t \geq 0
$$

Combining (64) and (65), we deduce that there exists $\Lambda>0$ for which the estimate

$$
\frac{d \mathfrak{L}(t)}{d t} \leq-\Lambda \mathfrak{L}(t), \quad \forall t \geq 0,
$$

holds. Integrating $(64)$ over $(0, t)$ once again, then (38) holds. Then, the proof is complete.

\section{ReFERENCES}

[1] C. Abdallah, P. Dorato, J. Benitez-Read and R. Byrne, Delayed positive feedback can stabilize oscillatory system, in: Proceedings of the 1993 American Control Conference, pp. 3106-3107, San Francisco, CA, USA, 1993.

[2] K. Ammari, S. Nicaise, C. Pignotti, Feedback boundary stabilization of wave equations with interior delay, Systems. Cont. Letters, 59: 623-628, 2010. 
[3] W. D. Bastos and C. A. Raposo, Transmission problem for waves with frictional damping, Electron. J. Differential Equations, Vol. 2007 (2007), No. 60, 1-10.

[4] A. Benseghir, Existence and exponential decay of solutions for transmission problems with delay, Electronic J. Diff. Equ., Vol. 2014 (2014), No. 212, pp. 1-11.

[5] R. Datko, Not all feedback stabilized hyperbolic systems are robust with respect to small time delays in their feedbacks, SIAM J. Control Optim., 26:697-713, 1988.

[6] R. Datko, Two questions concerning the boundary control of certain elastic systems, J. Differential Equations, 92 (1): 27-44, 1991.

[7] R. Datko, J. Lagnese, M. P. Polis, An example on the effect of time delays in boundary feedback stabilization of wave equations, SIAM J. Control Optim., 24(1): 152-156, 1986.

[8] T. Kato, Nonlinear semigroups and evolution equations, J. Math. Soc. Japan, 19:508$520,1967$.

[9] T. F. Ma, H. P. Oquendo, A transmission problem for beams on nonlinear supports, Bound. Value Probl., pages Art. ID 75107, 14, 2006.

[10] A. Marzocchi, J. E. Muñoz Rivera, M. G. Naso, Asymptotic behavior and exponential stability for a transmission problem in thermoelasticity, Math. Meth. Appl. Sci., 25: 955-980, 2002.

[11] A. Marzocchi, J. E. Muñoz Rivera, M. G. Naso, Transmission problem in thermoelasticity with symmetry, IMA Journal of Appl. Math., 63(1): 23-46, 2002.

[12] S. A. Messaoudi, B. Said-Houari, Energy decay in a transmission problem in thermoelasticity of type iii, IMA. J. Appl. Math, 74: 344-360, 2009.

[13] J. E. Muñoz Rivera, H. P. Oquendo, The transmission problem of viscoelastic waves, Acta Appl. Math., 62(1): 1-21, 2000.

[14] S. Nicaise, C. Pignotti, Stability and instability results of the wave equation with a delay term in the boundary or internal feedbacks, SIAM J. Control Optim., 45(5): 1561-1585, 2006.

[15] S. Nicaise, J. Valein and E. Fridman, Stability of the heat and of the wave equations with boundary time-varying delays, Discrete Contin. Dyn. Syst. Ser. S, 2: 559-581, 2009 .

[16] S. Nicaise and C. Pignotti, Interior feedback stabilisations of wave equations with time dependent delay, Elect. J. Deff. Eq. 41:2011 (2011), 01-20.

[17] S. Nicaise and C. Pignotti, Stabilization of the wave equation with boundary or internal distributed delay, Differential Integral Equations 21:9-10 (2008), 935-958. 
[18] I. H. Suh and Z. Bien, Use of time delay action in the controller design, IEEE Trans. Autom. Control 25:3 (1980), 600-603.

[19] C. Q. Xu, S. P. Yung, L. K. Li, Stabilization of the wave system with input delay in the boundary control, ESAIM: Control Optim. Calc. Var., 12: 770-785, 2006.

\section{Salah Zitouni}

Department of Mathematics and Informatics Univ Souk Ahras

P.O. Box 1553, Souk Ahras, 41000

Algeria

E-mail address: zitsala@yahoo.fr

\section{Abdelouaheb Ardjouni}

Department of Mathematics and Informatics Univ Souk Ahras

P.O. Box 1553, Souk Ahras, 41000

Algeria

E-mail address: abd_ardjouni@yahoo.fr

\section{Khaled Zennir}

Department of Mathematics

College of Sciences and Arts

Al-Ras, Al-Qassim University

Kingdom of SAudi Arabia

E-mail address: khaledzennir2@yahoo.com

\section{RACHIDA Amiar}

Department of Mathematics

FACUlty of Sciences

Univ AnNabA

P.O. Box 12, Annaba 23000

Algeria

E-mail address: rrachida_2000@yahoo.fr 